\title{
Olfactory and gustatory dysfunctions in 100 patients hospitalized for COVID-19: sex differences and recovery time in real-life
}

\author{
Simone Meini ${ }^{1}\left[\right.$ [ Lorenzo Roberto Suardi ${ }^{2} \cdot$ Michele Busoni $^{3} \cdot$ Anna Teresa Roberts $^{4} \cdot$ Alberto Fortini $^{5}$
}

Received: 25 April 2020 / Accepted: 29 May 2020 / Published online: 4 June 2020

(c) Springer-Verlag GmbH Germany, part of Springer Nature 2020

\begin{abstract}
Purpose COVID-19 displays a variety of clinical manifestations; in pauci-symptomatic patients olfactory (OD) and gustatory dysfunctions (GD) may represent the first or only symptom. This topic is currently arousing great interest, and a growing number of papers are being published. Aim of this study is to investigate the timing of recovery from OD and GD in a reallife population hospitalized for COVID-19.

Methods We followed up by a phone interview the first 100 patients discharged a month earlier from three Italian nonintensive care wards.

Results All 100 patients were Caucasian, mean age was 65 years, $60 \%$ were males. Forty-two patients (mean age 63 years) experienced subjective chemosensory dysfunctions (29 OD and $41 \mathrm{GD}$ ): the male/female ratio was 2:1; 83\% reported a complete or near complete recovery at follow-up. The recovery rate was not significantly different between males and females. The mean duration of OD and GD was 18 and 16 days, respectively. The mean recovery time from OD or GD resulted significantly longer for females than for males ( 26 vs 14 days, $P=0.009$ ). Among the 42 symptomatic, the mean age of males was significantly higher than that of females ( 66 vs 57 years, $P=0.04$ ), while the opposite was observed in the 58 asymptomatic patients (60 vs 73 years, $P=0.0018$ ).

Conclusions Recovery from OD or GD was rapid, occurring within 4 weeks in most patients. Chemosensory dysfunctions in women was less frequent, but longer lasting. The value of our study is its focus on a population of hospitalized patients significantly older than those previously described, and the additional data on gender differences.
\end{abstract}

Keywords COVID-19 $\cdot$ Ageusia $\cdot$ Anosmia $\cdot$ Recovery $\cdot$ Sex $\cdot$ Age

\section{Introduction}

Starting in December 2019 in Wuhan (Hubei province, China), a novel coronavirus, designated SARS-CoV-2, has caused an international outbreak of a respiratory illness

Simone Meini

simonemeini2@gmail.com

1 Internal Medicine Unit, Santa Maria Annunziata Hospital, Florence, Italy

2 Infectious Disease Unit, Santa Maria Annunziata Hospital, Florence, Italy

3 Otolaryngology-Head and Neck Surgery Unit, Santa Maria Nuova Hospital, Florence, Italy

4 Medical Department-Hospital Area, AUSL Toscana Nord-Ovest, Pisa, Italy

5 Internal Medicine Unit, Nuovo San Giovanni Di Dio Hospital, Florence, Italy
(COVID-19), rapidly evolving into a pandemic. Most cases are asymptomatic or self-limiting, but the clinical spectrum extends to severe progressive pneumonia with acute respiratory distress syndrome, a life-threatening condition requiring mechanical ventilation and intensive care support. A wide variety of clinical manifestations have been described, also involving the central nervous system [1]. In a not negligible number of patients, especially pauci-symptomatic ones, olfactory (OD) and gustatory dysfunctions (GD) may represent the first or only symptom. Data on chemosensory dysfunctions have been published only very recently, and the prevalence described is surprisingly variable, ranging from $5.1 \%$ and $5.6 \%$ in a Chinese cohort [1] to $86 \%$ and $88 \%$ in a European study [2], for OD and GD, respectively. Both studies relied on subjective reports of chemosensory dysfunction, rather than objective testing. The two populations in question were also very different regarding severity of illness and age: Mao and coworkers [1] studied a population 
of hospitalized patients with a mean age of 53 years, of which $41 \%$ presenting severe infection according to their respiratory status, Lechien's cases [2] were mainly mild-tomoderate, with a mean age of 37 years, so both relatively young populations, possibly non representative of real-life COVID-19 patients. Furthermore, the brief follow-up period in both studies, limited to the acute phase, did not allow to assess permanent anosmia or ageusia, and, to date, it is not well known whether recovery of olfactory and gustatory functions will be complete nor how long it will take.

The aim of our study is to investigate the timing of recovery from olfactory and gustatory dysfunctions in a cohort of COVID-19 patients hospitalized one month earlier in non-intensive care wards, an older population than that previously investigated in the early phases of the COVID-19 pandemic.

\section{Materials and methods}

In April 2020, we followed up the first 100 COVID-19 patients discharged a month earlier from three non-intensive care wards in Florence, Italy (Internal Medicine Units of the "Santa Maria Annunziata" and "Nuovo San Giovanni di Dio" hospitals, and Infectious Disease Unit of the "Santa Maria Annunziata" hospital). From the computerized database of these hospitals we identified all adults hospitalized and diagnosed as COVID-19 by reverse transcriptase-polymerase chain reaction (RT-PCR) testing for SARS-CoV-2 on nasopharyngeal swab. Clinical histories and the occurrence of neurological disorders during hospitalization were assessed from electronic medical charts. We telephoned each patient and asked if he/she had had, at any time of the disease, OD and/or GD, and if so, how many days it lasted. In particular, to assess OD and GD, patients were asked whether they had experienced any alterations or reductions of odor, flavor and taste since the onset of the illness. The five basic taste qualities (sweet, salty, bitter, sour, umami) were not singularly investigated.

In case of persistence, interviewees were asked to score the subjective function recovery on a 100-point scale, as previously done by Lee et al. [3]. We considered recovery as nearly complete when patients reported a recovery of 80-95 points and considered the loss not disabling and almost resolved; recovery was considered complete for 100 points.

We focused solely on olfactory and gustatory disorders, thus clinical presentation and comorbidities (except the assessment of pre-existing olfactory and gustatory dysfunctions) were not considered at the time of the survey. Patients with a history of cognitive impairment, unlikely to answer reliably, were excluded from follow-up study. Data were collected in a dedicated case record form.
Continuous variables were presented as mean values and the categorical variables were presented as counts and percentages. Analyses were mainly descriptive. We compared continuous variables by using Student's $t$ test proportions for categorical variables by using the $\chi^{2}$ test or Fisher exact test. We considered a $P$ value below 0.05 to be statistically significant. We used for all analyses MedCalc ${ }^{\circledR}$ statistical software version 16.4.3 (Ostend, Belgium).

\section{Results}

All 100 patients responded to the survey. All patients were Caucasian, mean age ( \pm standard deviation) was $65 \pm 15$ years (range 29-94 years), and 60\% were males. No patient was reported to suffer from chronic sinonasal disorders already causing chemosensory dysfunctions before the index hospitalization. No patient presented any relevant neurological disorder (excluding headache) during hospitalization.

In the group reporting no dysfunction (58\% of patients) the mean age was 66 years.

Forty-two patients, with a mean age of 63 years, reported chemosensory dysfunctions: 41 patients reported GD, 29 OD, 28 both GD and OD. All patients complained of their symptoms starting very early, at the most within one week the first noted symptoms. Out of these 42 symptomatic patients, 28 were males, thus the male/female ratio was $2: 1$. Of the 42 symptomatic patients, $64 \%$ reported a complete and $19 \%$ a near complete recovery, thus $83 \%$ reported an almost resolved dysfunction at follow-up. The recovery rate was not significantly different between males and females. The mean duration of OD and GD in the entire population of symptomatic was 18 and 16 days, respectively. The mean recovery time from OD or GD resulted significantly longer for females than for males (26 vs 14 days, $P=0.009$ ).

Among the 42 symptomatic patients, the mean age of males was significantly higher than that of females (66 vs 57 years, $P=0.04$ ), while the opposite was observed in the 58 asymptomatic patients (60 vs 73 years, $P=0.0018$ ).

A partial recovery (30-50 points) from GD was reported by $4 / 42$ patients after a mean of 32 days, in 2 cases with associated OD. Three patients reported no improvement of both ageusia and anosmia after a mean of 27 days.

Table 1 shows the demographical characteristics and the recovery rate and time observed in our population.

\section{Discussion and conclusions}

In our experience, among a population of hospitalized COVID-19 patients aged 65 years, $42 \%$ complained of olfactory (anosmia or hyposmia) or gustatory (ageusia or 
Table 1 Sex differences and recovery rate and time in 100 hospitalized COVID-19 patients

\begin{tabular}{|c|c|c|c|c|c|c|c|c|c|c|}
\hline $\begin{array}{l}\text { COVID-19 } \\
\text { Groups }\end{array}$ & \multicolumn{2}{|c|}{$\begin{array}{c}\text { All population } \\
(n=100)\end{array}$} & \multicolumn{2}{|c|}{$\begin{array}{c}\text { OD or GD } \\
(n=42)\end{array}$} & \multicolumn{2}{|c|}{$\begin{array}{c}\text { OD } \\
(n=29)\end{array}$} & \multicolumn{2}{|c|}{$\begin{array}{c}\text { GD } \\
(n=41)\end{array}$} & \multicolumn{2}{|c|}{$\begin{array}{c}\text { No dysfunction } \\
(n=58)\end{array}$} \\
\hline $\begin{array}{l}\text { Sex } \\
(n=\text { number pts) }\end{array}$ & $\begin{array}{l}\text { Males } \\
(n=60)\end{array}$ & $\begin{array}{c}\text { Females } \\
(n=40)\end{array}$ & $\begin{array}{l}\text { Males } \\
(n=28)\end{array}$ & $\begin{array}{c}\text { Females } \\
(n=14)\end{array}$ & $\begin{array}{l}\text { Males } \\
(n=18)\end{array}$ & $\begin{array}{c}\text { Females } \\
(n=11)\end{array}$ & $\begin{array}{l}\text { Males } \\
(n=28)\end{array}$ & $\begin{array}{c}\text { Females } \\
(n=13)\end{array}$ & $\begin{array}{l}\text { Males } \\
(n=32)\end{array}$ & $\begin{array}{c}\text { Females } \\
(n=26)\end{array}$ \\
\hline Age, mean, years & 63 & 67 & 66 & 57 & 66 & 57 & 66 & 57 & 60 & 73 \\
\hline$P$ value & \multicolumn{2}{|c|}{$P=0.16$} & \multicolumn{2}{|c|}{$P=0.04$} & \multicolumn{2}{|c|}{$P=0.12$} & \multicolumn{2}{|c|}{$P=0.04$} & \multicolumn{2}{|c|}{$P=0.0018$} \\
\hline $\begin{array}{l}\text { Recovery rate (80- } \\
100 \text { points), } n(\%)\end{array}$ & - & - & $25(89)$ & $10(71)$ & $16(89)$ & $8(73)$ & $25(89)$ & $9(69)$ & NA & NA \\
\hline$P$ value & \multicolumn{2}{|c|}{-} & \multicolumn{2}{|c|}{$P=0.12$} & \multicolumn{2}{|c|}{$P=0.33$} & \multicolumn{2}{|c|}{$P=0.18$} & \multicolumn{2}{|c|}{ NA } \\
\hline $\begin{array}{l}\text { Recovery time, } \\
\text { mean, days }\end{array}$ & - & - & 14 & 26 & 16 & 25 & 14 & 27 & \multicolumn{2}{|c|}{ NA } \\
\hline$P$ value & \multicolumn{2}{|c|}{ - } & \multicolumn{2}{|c|}{$P=0.009$} & \multicolumn{2}{|c|}{$P=0.07$} & \multicolumn{2}{|c|}{$P=0.0021$} & \multicolumn{2}{|c|}{ NA } \\
\hline
\end{tabular}

$O D$ olfactory dysfunction, $G D$ gustatory dysfunction, $N A$ not applicable

dysgeusia) dysfunctions: this percentage resulted half of that reported by Lechien et al. [2] in a younger population, but higher than that reported by Mao et al. [1] in their hospitalized patients.

OD and GD were reported, respectively, by $29 \%$ and $41 \%$ of our patients, almost always occurring in the earlier stages of the disease: the lower incidence of smell over taste disturbances has been reported also by Klopfenstein et al. [4] ( $47 \%$ vs $85 \%$ ), and, as previously mentioned, by Mao et al. [1] and Lechien et al. [2].

Females have been reported $[2,4]$ as significantly more affected by these dysfunctions, but our study, in which females accounted for $40 \%$ of total population (a percentage which reflects the documented male predisposition for COVID-19 [5]), did not confirm this data. In fact, most women $(65 \%)$ did not report any chemosensory symptom.

In our cohort, recovery from OD/GD was rapid, occurring within 4 weeks in most patients, and the mean recovery time from OD or GD resulted significantly longer for females than for males ( 26 vs 14 days, $P=0.009$ ).

Lechien et al. [2] already reported an early olfactory recovery rate of $44 \%$, occurring within the first 8 days following the resolution of the disease in $73 \%$ of patients with OD. Klopfenstein et al. [4] reported a mean duration of anosmia of 9 days, with a complete recovery occurring in almost all patients within 4 weeks.

In our study chemosensory dysfunctions in women was less frequent, but longer lasting.

In our cohort of patients admitted for a life-threatening illness, none was found to report chronic sinonasal disorders already causing chemosensory dysfunctions before the hospitalization, but it is likely that among the clinical information initially collected there were some missing data, since chronic rhinosinusitis is in fact a relevant health problem affecting $5-12 \%$ of the general population [6]. The same consideration applies to any previous sinonasal surgery.
Our observations derive from a small sample of patients: they confirm in part what has recently been reported about these dysfunctions by other authors, in particular the rapid recovery time. The value of our study is its focus on a hospitalized population significantly older than those previously described, more representative of real-life COVID-19 epidemiology, and to further investigate gender differences.

One limitation of our study was that patients did not undergo specific examinations for chemosensory functions, such as psychophysical and/or electrophysiological tests, but all non-essential procedures were purposefully avoided to reduce the risk of cross infection.

Moreover, our patients were only asked if they had had an altered or reduced perception of odors, flavor and taste compared to their pre-disease status. Terms such as "taste", "flavor", "smell", "odor", express different aspects of very complex sensory experiences, characterized by a significant subjective component; some languages do not have specific terms for each [7]. For these reasons, we decided to collect "coarser" data, but for which our patients had no difficulty in identifying incidence or persistence.

COVID-19 patients need yet to be tested for gustatory impairment, assuming it could also be just the sense of smell being impaired in terms of reduced "flavor" perception: further studies are needed to specify impairments by testing for ortho-retronasal olfactory, and gustatory dysfunctions [8].

Most of our patients, even without having been admitted to an intensive care unit for invasive ventilation, experienced severe acute respiratory failure: it is possible that chemosensory impairment could at least partly be considered as a consequence of the impaired respiratory function $[9,10]$.

Smell and taste loss have been reported as being significantly higher in COVID-19 patients versus non-COVID-19 patients with influenza-like symptoms (68\% and $71 \%$ vs 16 and $17 \%$, respectively) [11]. Moreover, the rapid recovery observed in COVID-19 differs from other post-viral OD, for 
which over $80 \%$ of the patients reported recovery after one year and longer [3], suggesting a slow regeneration of the olfactory epithelium and olfactory bulb. The damage SARSCoV-2 inflicts on taste and smell must be different from that of other viruses, but the pathophysiological mechanisms are largely unknown. Gilani et al. [12] hypothesized that the mechanism of injury is similar to that of other coronaviruses infections that cause central and peripheral neurologic deficits, but we agree with Vaira et al. [13] when they suggest that, given the high rate of rapid recovery, it is reasonable to hypothesize that the OD is not related to definitive damage from the virus to the neuronal cells, and a damage to other cell types appears more likely. In case of SARS-CoV-2-induced anosmia, magnetic resonance imaging of the olfactory bulb did not show abnormal findings regarding its volume or signal intensity [14]. Moreover, the rapid recovery leads us to hypothesize the role of a biochemical mediator. The question is: what could it be? We can only speculate.

One clue could be that angiotensin-converting-enzyme (ACE)-inhibitors can cause OD [15]. SARS-CoV-2 enters host cells by binding (then down-regulating it) the ACE2 receptor [16]: ACE2 receptor and TMPRSS2 proteases, crucial for S protein priming, are both expressed by nonneuronal cells of the olfactory epithelium [17], and ACE2 is diffusely expressed on the mucous membrane of the oral cavity, particularly on the tongue [18]. The reduced activity of ACE and ACE2 leads to increased levels of bradykinin (BK) and [des-Arg9]-BK, respectively: these kinins, through their receptors $\mathrm{B} 2 \mathrm{R}$ and $\mathrm{B} 1 \mathrm{R}$, could mediate many features observed in COVID-19, such as dry cough, inflammation, pain, vasodilation, and vascular permeability. BK seems also involved in GD, through a neurogenic inflammatory mechanism or an excess of activity directly in the central nervous system [19], and it is interesting to note that BK can also mediate a painful warm sensation [19] trough the ion channel TRPA1 on trigeminal neurons that project to the tongue [20]: it is known that some COVID-19 patients also reported a burning mouth symptom. Finally, B2R has been described in the olfactory bulb [21], where it obviously has a role to play. In COVID-19 patients, olfactory dysfunction is not associated with rhinorrhea or nasal obstruction [2], but for the above-mentioned reasons, we do believe that the role of BK cannot be excluded in determining olfactory and/or gustatory dysfunctions.

We still have a long way to go in understanding the pathogenesis of these COVID-19-associated symptoms, and we hope for more research in this field, not least because it could help to better clarify the mechanisms leading to life-threatening organ damage. Finally, we agree with those [22] who recommend that, pending definitive evidence, in the context of the current pandemic, any patient with a new isolated olfactory or gustatory dysfunction should be considered as being infected with SARS-CoV-2 until proven otherwise.
Funding None.

\section{Compliance with ethical standards}

Conflict of interest The authors declare that they have no conflict of interest.

\section{References}

1. Mao L, Jin H, Wang M, Hu Y, Chen S, He Q et al (2020) Neurologic manifestations of hospitalized patients with coronavirus disease 2019 in Wuhan, China. JAMA Neurol. https://doi. org/10.1001/jamaneurol.2020.1127

2. Lechien JR, Chiesa-Estomba CM, De Siati DR, Horoi M, Le Bon SD, Rodriguez A et al (2020) Olfactory and gustatory dysfunctions as a clinical presentation of mild-to-moderate forms of the coronavirus disease (COVID-19): a multicenter European study. Eur Arch Otorhinolaryngol. https://doi.org/10.1007/s00405-02005965-1

3. Lee DY, Lee WH, Wee JH, Kim JW (2014) Prognosis of postviral olfactory loss: follow-up study for longer than one year. Am J Rhinol Allergy 28(5):419-422

4. Klopfenstein T, Kadiane-Oussou NJ, Toko L, Royer PY, Lepiller Q, Gendrin V, Zayet S (2020) Features of anosmia in COVID-19. Med Mal Infect. https://doi.org/10.1016/j.medmal.2020.04.006

5. Cai H (2020) Sex Difference and Smoking Predisposition in Patients With COVID-19. Lancet Respir Med. Accessed 2020 Mar 11 [Online ahead of print]

6. Fokkens WJ, Lund VJ, Hopkins C, Hellings PW, Kern R, Reitsma $S$ et al (2020) European position paper on rhinosinusitis and nasal polyps 2020. Rhinology 58(S29): 1-464

7. Rozin P (1982) Taste-smell confusions" and the duality of the olfactory sense. Percept Psychophys 31(4):397-401

8. Besser G, Tianxiang Liu D, Prem B, Iabloncsik D, Pablik E, Mueller CA, Renner B (2020) Retronasal olfactory testing using candies sent by post and for screening purposes: a feasibility study. Rhinology. https://doi.org/10.4193/Rhin19.230

9. Günther R, Schrempf W, Hähner A, Hummel T, Wolz M, Storch A, Hermann A (2018) Impairment in respiratory function contributes to olfactory impairment in amyotrophic lateral sclerosis. Front Neurol 9:79. https://doi.org/10.3389/fneur.2018.00079

10. Kaya KS, Akpınar M, Turk B, Seyhun N, Cankaya M, Coskun BU (2020) Olfactory function in patients with obstructive sleep apnea using positive airway pressure. Ear Nose Throat J 99(4):239-244

11. Yan CH, Faraji F, Prajapati DP, Boone CE, DeConde AS (2020) Association of chemosensory dysfunction and Covid-19 in patients presenting with influenza-like symptoms. Int Forum Allergy Rhinol. https://doi.org/10.1002/alr.22579

12. Gilani S, Roditi R, Naraghi M (2020) COVID-19 and anosmia in Tehran. Iran Med Hypotheses 141:109757. https://doi. org/10.1016/j.mehy.2020.109757

13. Vaira LA, Salzano G, Fois AG, Piombino P, De Riu G (2020) Potential pathogenesis of ageusia and anosmia in COVID-19 patients. Int Forum Allergy Rhinol. https://doi.org/10.1002/ alr.22593

14. Galougahi MK, Ghorbani J, Bakhshayeshkaram M, Naeini AS, Haseli S (2020) Olfactory bulb magnetic resonance imaging in SARS-CoV-2-induced anosmia: the first report. Acad Radiol. https://doi.org/10.1016/j.acra.2020.04.002

15. Scangas GA, Bleier BS (2017) Anosmia: differential diagnosis, evaluation, and management. Am J Rhinol Allergy 31:e3-7

16. Hoffmann M, Kleine-Weber H, Schroeder S, Krüger N, Herrler T, Erichsen S et al (2020) SARS-CoV-2 cell entry depends on 
ace 2 and tmprss 2 and is blocked by a clinically proven protease inhibitor. Cell 181(2):271-280

17. Butowt R, Bilinska K (2020) SARS-CoV-2: olfaction, brain infection, and the urgent need for clinical samples allowing earlier virus detection. ACS Chem Neurosci. https://doi.org/10.1021/ acschemneuro.0c00172

18. Xu H, Zhong L, Deng J, Peng J, Dan H, Zeng X et al (2020) High expression of ACE2 receptor of 2019-nCoV on the epithelial cells of oral mucosa. Int J Oral Sci 12(1):8

19. Belowska-Bień K, Szeląg E, Szeląg J, Skrzypiec-Spring M (2017) Bradykinin—an undervalued mediator? Post N Med 8:440-446
20. Bandell M, Story GM, Hwang SW, Viswanath V, Eid SR, Petrus MJ et al (2004) Noxious cold ion channel TRPA1 is activated by pungent compounds and bradykinin. Neuron 41(6):849-857

21. Chen EY, Emerich DF, Bartus RT, Kordower JH (2000) B2 bradykinin receptor immunoreactivity in rat brain. J Comp Neurol 427(1):1-18

22. Reinhard A, Ikonomidis C, Broome M, Gorostidi F (2020) Anosmia and COVID-19. Rev Med Suisse 16:849-851

Publisher's Note Springer Nature remains neutral with regard to jurisdictional claims in published maps and institutional affiliations. 